\title{
Topologically Massive Yang-Mills field on the Null-Plane: A Hamilton-Jacobi approach
}

\author{
M. C. Bertin*, B. M. Pimentel*, C. E. Valcárcel* and G. E. R. Zambrano ${ }^{\dagger}$ \\ *Instituto de Física Teórica, UNESP - São Paulo State University, Caixa Postal 70532-2, \\ 01156-970, São Paulo, SP, Brazil \\ ${ }^{\dagger}$ Departamento de Física, Universidad de Nariño, Calle 18 Carrera 50, San Juan de Pasto, Nariño,
} Colombia

\begin{abstract}
.
Non-abelian gauge theories are super-renormalizable in $2+1$ dimensions and suffer from infrared divergences. These divergences can be avoided by adding a Chern-Simons term, i.e., building a Topologically Massive Theory. In this sense, we are interested in the study of the Topologically Massive Yang-Mills theory on the Null-Plane. Since this is a gauge theory, we need to analyze its constraint structure which is done with the Hamilton-Jacobi formalism. We are able to find the complete set of Hamiltonian densities, and build the Generalized Brackets of the theory. With the GB we obtain a set of involutive Hamiltonian densities, generators of the evolution of the system.
\end{abstract}

Keywords: Hamilton-Jacobi formalism, Topologically Massive Yang-Mills

PACS: $11.15 . \mathrm{Wx}$

\section{INTRODUCTION}

Since the work of Witten [1], Topological Quantum Field Theories (TQFT) have been used in different branches of modern physics, from condensed matter to string theory. Chern-Simons (CS) gauge theory is a Schwarz type TQFT defined in odd dimensions, i.e., its Lagrangian density does not have an explicit dependence on the metric of spacetime. Along with the gauge invariance, its classical action is invariant under the change of the background metric.

Topologically Massive (TM) theories were introduced by Deser [2]. These theories are built adding a CS term to a three dimensional kinetic action. Thus we have the Maxwell-Chern-Simons Theory (MCS), the Topologically Massive Yang-Mills theory (TMYM) and Topologically Massive Gravity (TMG). As a result of adding the CS term, it is provided mass for the gauge fields. Another important feature of TM gauge theories is that at quantum level the topological mass provides an infrared cut-off.

TM theories, as any other gauge theories, have a local invariance which leads to constraints [3]. The Dirac's canonical formalism [4] is used extensively to analyze constrained systems. This formalism has been used to study the TMYM theory in [5].

Güller [6] has developed the Hamilton-Jacobi (HJ) formalism as an alternative method for study constrained systems. This method is a generalization of the work of Caratheodory [7]. Since this work several improvements have been done [8]. The HJ formalism is characterized by a set of Hamilton-Jacobi Partial Differential Equations (HJPDE) also known as Hamiltonian densities. The integrability of this system is achieved if the Frobenius' Integrability Condition (IC) is satisfied. 
In this work we apply the HJ formalism to analyze the constraint structure of the TMYM theory on the null-plane. We build its Generalized Brackets (GB) and obtain the characteristic equations.

\section{THE TMYM THEORY ON THE NULL-PLANE}

The TMYM theory is described by the following gauge invariant action

$$
I=\int d^{3} x \mathscr{L}=\int d^{3} x\left\{-\frac{1}{4} F^{a \mu v} F_{\mu \nu}^{a}-\frac{\mu}{4} \varepsilon^{\alpha \beta \gamma}\left[F_{\alpha \beta}^{a} A_{\gamma}^{a}-\frac{g}{3} f^{a b c} A_{\alpha}^{a} A_{\beta}^{b} A_{\gamma}^{c}\right]\right\},
$$

where $\mu$ is the mass parameter, and $F_{\alpha \beta}^{a} \equiv \partial_{\alpha} A_{\beta}^{a}-\partial_{\beta} A_{\alpha}^{a}+g f^{a b c} A_{\alpha}^{b} A_{\beta}^{c}$.

We proceed to describe the dynamical evolution of the system with the time variable $\tau \equiv x^{+}$, which is a light-cone coordinate defined in $(2+1)$ dimensions as

$$
x^{+} \equiv \frac{1}{\sqrt{2}}\left(x^{0}+x^{2}\right), \quad x^{-} \equiv \frac{1}{\sqrt{2}}\left(x^{0}-x^{2}\right), \quad x^{1}=x^{1} .
$$

In the Light-Cone coordinates, some of the conjugated momenta are given by

$$
\pi_{a}^{+}=0, \pi_{a}^{1}=F_{-1}^{a}+\frac{\mu}{2} A_{-}^{a} .
$$

These are constraints of the theory. The momentum $\pi^{-}$gives the dynamical relation

$$
\partial_{+} A_{-}^{a}=\pi_{a}^{-}+\partial_{-} A_{+}^{a}-g f^{a b c} A_{+}^{b} A_{-}^{c}+\frac{\mu}{2} A_{1}^{a} .
$$

With the presence of constraints, we build the canonical Hamiltonian density

$$
\mathscr{H}_{\tau}=\frac{1}{2}\left[\pi_{a}^{-}+\frac{\mu}{2} A_{1}^{a}\right]^{2}-A_{+}^{a}\left[D_{-} \pi_{a}^{-}+D_{1} \pi_{a}^{1}+\frac{\mu}{2}\left(\partial_{1} A_{-}^{a}-\partial_{-} A_{1}^{a}\right)\right],
$$

where we define the covariant derivative $D_{\mu} X^{a}=\partial_{\mu} X^{a}-g f^{a b c} X^{b} A_{\mu}^{c}$.

The HJ formalism is characterized by the existence of a function $S$ such that $\pi_{a}^{\mu}=$ $\partial S / \partial A_{\mu}^{a}$. Therefore, eqs. (3) are actually partial differential equations. Together with the usual HJ equation, they form a set of HJ partial differential equations (HJPDE), also called Hamiltonian densities, given by

$$
\begin{aligned}
\mathscr{H}^{\prime \tau} & =\pi^{\tau}+\mathscr{H}_{\tau}=0, \\
\mathscr{H}_{a}^{\prime+} & =\pi_{a}^{+}=0, \\
\mathscr{H}_{a}^{\prime 1} & =\pi_{a}^{1}-F_{-1}^{a}-\frac{\mu}{2} A_{-}^{a}=0,
\end{aligned}
$$

where we identify $\tau=x^{+}, A_{+}^{a}$ and $A_{1}^{a}$ as parameters of the theory, each one related to a respective Hamiltonian $\mathscr{H}^{\prime \tau}, \mathscr{H}_{a}^{\prime+}$ and $\mathscr{H}_{a}^{\prime 1}$. Then, we write the fundamental differential as

$$
d F=\int d y^{2}\left[\left\{F, \mathscr{H}^{\prime \tau}(y)\right\} d \tau+\left\{F, \mathscr{H}_{a}^{\prime+}(y)\right\} d A_{+}^{a}(y)+\left\{F, \mathscr{H}_{a}^{\prime 1}(y)\right\} d A_{1}^{a}(y)\right] .
$$


When imposing the IC, we obtain a complete set of Hamiltonian densities. Since

$$
d \mathscr{H}_{a}^{\prime+}=\left[D_{-} \pi_{a}^{-}+D_{1} \pi_{a}^{1}+\frac{\mu}{2}\left(\partial_{1} A_{-}^{a}-\partial_{-} A_{1}^{a}\right)\right] d x^{+},
$$

we define a new Hamiltonian

$$
\mathscr{C}_{a} \equiv D_{-} \pi_{a}^{-}+D_{1} \pi_{a}^{1}+\frac{\mu}{2}\left(\partial_{1} A_{-}^{a}-\partial_{-} A_{1}^{a}\right)
$$

This new Hamiltonian, along with $\mathscr{H}_{a}^{\prime 1}$, satisfy the IC $d \mathscr{C}_{a}^{\prime}=0$ and $d \mathscr{H}_{a}^{\prime 1}=0$ identically.

With the complete set of Hamiltonian densities we expand the phase space and promote $\omega^{a}$ as a variable of the theory. This parameter is related to the new Hamiltonian density. Then, the extended fundamental differential is given by

$$
d F=\int d^{2} y\left[\left\{F, \mathscr{H}^{\prime \tau}\right\} d \tau+\left\{F, \mathscr{H}_{a}^{\prime+}\right\} d A_{+}^{a}+\left\{F, \mathscr{H}_{a}^{\prime 1}\right\} d A_{1}^{a}+\left\{F, \mathscr{C}^{\prime a}\right\} d \omega_{a}\right] .
$$

At this stage, we separate the Hamiltonian densities others than $\mathscr{H}^{\prime \tau}$ as involutive or non-involutive. This allows us to reduce the phase space of the theory. We notice that $\mathscr{H}_{a}^{\prime 1}$ are the non-involutive densities since

$$
M_{a b}(x, y) \equiv\left\{\mathscr{H}_{a}^{\prime 1}(x), \mathscr{H}_{b}^{\prime 1}(y)\right\}=-2 D_{-}^{x} \delta_{a b} \delta(x-y) .
$$

The $\mathrm{HJ}$ formalism allows to eliminate the variables related to this constraints, $A_{1}^{a}$, as the evolution parameter of the system. This can be done if we define the GB

$$
\begin{aligned}
\{A(x), B(y)\}^{*}= & \{A(x), B(y)\} \\
& -\int d z \int d w\left\{A(x), \mathscr{H}_{r}^{\prime 1}(z)\right\} G^{r s}(z, w)\left\{\mathscr{H}_{s}^{\prime 1}(w), B(y)\right\},
\end{aligned}
$$

where $G^{r s}(x, y)$ is the inverse matrix of $M_{a b}(x, y)$.

We obtain the following fundamental GB

$$
\begin{aligned}
& \left\{A_{\mu}^{a}(x), A_{v}^{b}(y)\right\}^{*}=\delta_{\mu}^{1} \delta_{v}^{1} G^{a b}(x, y) \\
& \left\{A_{\mu}^{a}(x), \pi_{b}^{v}(y)\right\}^{*}=\delta_{a b} \delta_{\mu}^{v} \delta(x-y)-\delta_{\mu}^{1}\left[\delta_{1}^{v} D_{-}^{y}-\delta_{-}^{v} D_{1}^{y}-\frac{\mu}{2} \delta_{-}^{v}\right] G^{a b}(x, y) \\
& \left\{\pi_{a}^{\mu}(x), \pi_{b}^{v}(y)\right\}^{*}=\left[\delta_{1}^{v} D_{-}^{y}-\delta_{-}^{v} D_{1}^{y}-\frac{\mu}{2} \delta_{-}^{v}\right]\left[\delta_{1}^{\mu} D_{-}^{x}-\delta_{-}^{\mu} D_{1}^{x}-\frac{\mu}{2} \delta_{-}^{\mu}\right] G^{a b}(x, y)(1)
\end{aligned}
$$

Under the GB, the evolution of the system does not depend on the variables related to the Hamiltonian with which the matrix $M^{a b}(x, y)$ is buildt, says $A_{1}^{a}$. The new fundamental differential is given by

$$
d F=\int d y^{2}\left[\left\{F, \mathscr{H}^{\prime \tau}(y)\right\}^{*} d \tau+\left\{F, \mathscr{H}_{a}^{\prime+}(y)\right\}^{*} d A_{+}^{a}(y)+\left\{F, \mathscr{C}^{\prime a}(y)\right\}^{*} d \omega_{a}(y)\right] .
$$


From where we obtain the characteristic equations

$$
\begin{aligned}
& \partial_{+} A_{-}^{a}=\pi_{a}^{-}+\partial_{-} A_{+}^{a}-g f^{a b c} A_{+}^{b} A_{-}^{c}+\frac{\mu}{2} A_{1}^{a}-D_{-} \partial_{+} \omega^{a}, \\
& \partial_{+} \pi_{a}^{+}=D_{-} \pi_{a}^{-}+D_{1} \pi_{a}^{1}+\frac{\mu}{2}\left(\partial_{1} A_{-}^{a}-\partial_{-} A_{1}^{a}\right), \\
& \partial_{+} \pi_{a}^{1}=-\frac{1}{2} D_{1} F_{+-}^{a}+\frac{\mu}{2} D_{-} A_{+}^{a}-\frac{\mu}{2} D_{-} \partial_{+} \omega^{a},
\end{aligned}
$$

along with the indeterminacy of $\partial_{+} A_{+}^{a}$.

\section{FINAL REMARKS}

In this work we presented the classical analysis of the constrained TMYM theory on the Null-Plane. The IC allows us to find the complete set of Hamiltonian densities of the theory and, after building the GB, we have reduced the phase space and identified the independent parameters of the theory.

In a future work we intend to do a comparative study of TMYM theory, using both the conventional $x^{0}$ and also using $x^{+}$time parameter. We also intend to show the relation between the involutive Hamiltonian densities and the generators of gauge transformation. under the scope of the HJ formalism.

\section{ACKNOWLEDGMENTS}

M. C. Bertin and C. E. Valcárcel were supported by CAPES. B. M. Pimentel was partially supported by CNPq.

\section{REFERENCES}

1. E. Witten, Commun. Math. Phys. 117, 353 (1988).

2. S. Deser, R. Jackiw and S. Templeton, Phys. Rev. Lett. 48, 975 (1982); Ann. Phys. 140, 372 (1982).

3. J. L. Anderson, Phys. Rev. 111, 965 (1958).

4. P. A. M. Dirac, Canad. J. Math. 2, 129 (1950); Canad. J. Math. 3, 1 (1951); Proc. Roy. Soc. A 246, 326 (1958); Lectures on Quantum Mechanics, Yeshiva University, New York, 1964.

5. D. Evens, G. Kunstatter, Phys. Rev. D 37, 435 (1988).

6. Y. Güler, Il Nuovo Cimento B 100, 251 (1987); J. Math. Phys. 30, 785 (1992); Il Nuovo Cimento B 107, 1398 (1992).

7. C. Carathéodory, Calculus of Variations and Partial Differential Equations of the First Order, American Mathematical Society; 3rd edition (1999).

8. B. M. Pimentel, R. G. Teixeira, Il Nuovo Cimento B 111, 841 (1996); B. M. Pimentel, R. G. Teixeira, Il Nuovo Cimento B 113, 805 (1998); B. M. Pimentel, R. G. Texeira, J. L. Tomazelli, Ann. Phys 267, 75 (1998); B. M. Pimentel, P. J. Pompeia, J.F. da Rocha-Neto, R.G. Teixeira, Gen. Rel. Grav. 35, 877 (2003); M. C. Bertin, B. M. Pimentel, P. J. Pompeia, Mod. Phys. Lett. A 20, 2873 (2005); B. M. Pimentel, P. J. Pompeia, J. F. da Rocha-Neto, Il Nuovo Cimento B 120, 981 (2005); M. C. Bertin, B. M. Pimentel, P. J. Pompeia, Ann. Phys. 323, 527 (2008); M. C. Bertin, B. M. Pimentel, C. E. Valcárcel, Ann. Phys. 323, 3137 (2008). 
Copyright of AIP Conference Proceedings is the property of American Institute of Physics and its content may not be copied or emailed to multiple sites or posted to a listserv without the copyright holder's express written permission. However, users may print, download, or email articles for individual use. 